\title{
ON THRESHOLD STRATEGIES AND THE SMOOTH-FIT PRINCIPLE FOR OPTIMAL STOPPING PROBLEMS
}

\author{
STÉPHANE VILLENEUVE, ${ }^{*}$ Université de Toulouse 1
}

\begin{abstract}
In this paper we investigate sufficient conditions that ensure the optimality of threshold strategies for optimal stopping problems with finite or perpetual maturities. Our result is based on a local-time argument that enables us to give an alternative proof of the smoothfit principle. Moreover, we present a class of optimal stopping problems for which the propagation of convexity fails.
\end{abstract}

Keywords: Optimal stopping; principle of smooth fit; local time

2000 Mathematics Subject Classification: Primary 60G40; 60J60

\section{Introduction}

One of the simplest formulations of stochastic optimal control is that of the optimal stopping of a one-dimensional diffusion process, where the only decision to be made is when to stop the process. In this paper we will consider continuous-time optimal stopping, as follows. Consider a state process modeled by the one-dimensional diffusion process $X_{t}$. We assume that the payoff from stopping at time $t$ is $g\left(X_{t}\right)$ and that the decision-maker wants to maximize the expected present value by choosing a stopping time. This may be modeled using two optimal stopping problems with respective value functions

$$
V(x)=\sup _{\tau \in \mathcal{T}_{0, \infty}} \mathrm{E}\left(\mathrm{e}^{-r \tau} g\left(X_{\tau}^{x}\right)\right)
$$

and

$$
V_{T}(x)=\sup _{\tau \in \mathcal{T}_{0, T}} \mathrm{E}\left(\mathrm{e}^{-r \tau} g\left(X_{\tau}^{x}\right)\right),
$$

where $\mathcal{T}_{0, \infty}$ and $\mathcal{T}_{0, T}$ are the sets of all admissible stopping times with values in $[0, \infty]$ and, respectively, $[0, T]$. The value function $V$ corresponds to a perpetual-maturity optimal stopping problem while the value function $V_{T}$ corresponds to an optimal stopping problem with finite maturity. In this paper we will focus our study on the perpetual-maturity case, but the corresponding findings in the finite-maturity case will be highlighted as the paper proceeds.

One major problem in the perpetual-maturity case is to prove the existence of an optimal stopping time $\tau^{*}$ satisfying

$$
V(x)=\mathrm{E}\left(\mathrm{e}^{-r \tau^{*}} g\left(X_{\tau^{*}}^{x}\right)\right) .
$$

According to optimal stopping theory (see [8]), we introduce the following sets, usually called the stopping region and the continuation region, respectively:

$$
E=\{x>0: V(x)=g(x)\}, \quad C=\{x>0: V(x)>g(x)\} .
$$

Received 6 April 2005; revision received 24 October 2006.

* Postal address: Manufacture des Tabacs, Université de Toulouse 1, 21 Allée de Brienne, 31000 Toulouse, France.

Email address: stephane.villeneuve@univ-tlse1.fr 
Under integrability conditions that will be made precise later, we have

$$
V(x)=\mathrm{E}\left(\mathrm{e}^{-r \tau_{E}} g\left(X_{\tau_{E}}^{x}\right)\right)
$$

where

$$
\tau_{E}=\inf \left\{t>0: X_{t} \in E\right\} .
$$

Frequently, in the case where $g$ is convex and nondecreasing, it is optimal to stop when the process $X_{t}$ exceeds some fixed threshold $x^{*}$, called the optimal exercise boundary; then $E=$ $\left[x^{*}, \infty\right)$. In this case we say that a threshold strategy is optimal. Once the optimality of a threshold strategy has been proved, two methods are commonly used to compute the value function. The first is the free boundary problem approach, where the value function can be computed explicitly using a free boundary formulation and the principle of smooth fit, i.e. the value function is differentiable across the optimal exercise boundary $x^{*}$ (see [20, Chapter 10, Section 4] and the references therein). The second approach is based on fluctuation theory (see [16]) and relies on the computation of the Laplace transform of the hitting time at level $x^{*}$. Furthermore, Dupuis and Wang [6] identified the rate of convergence from discrete-time to continuous-time optimal stopping problems under the assumption that the stopping region is the interval $\left[x^{*}, \infty\right)$. Hence, threshold strategies are among the most important and common strategies for solving one-dimensional optimal stopping problems.

However, in recent papers, Dayanik and Karatzas [3, Section 6.4] and Décamps et al. [4] gave very simple examples of convex, nondecreasing payoffs for which the optimal stopping strategy is not of threshold type. This leads to the question of main interest: can we find a set of easily testable conditions ensuring the existence of an optimal threshold strategy for a general state process?

A first step has been made by Jonsson et al. [12], who gave results for optimal stopping problems in discrete time. In this paper we will give results for continuous-time optimal stopping problems that are linked to the characteristic operator $\mathcal{L}$ such that

$$
\mathcal{L} u=\frac{1}{2} x^{2} \sigma(x)^{2} u^{\prime \prime}+x(r-\delta(x)) u^{\prime} .
$$

The outline of the paper is as follows. In Section 2 we introduce our notation and define the basic assumptions of the continuous-time optimal stopping problem. We will give conditions necessary and sufficient to ensure the nonemptiness of the stopping region in Section 3. Section 4 is devoted to our main results. We state a condition sufficient to ensure the optimality of a threshold strategy and give an alternative proof that the smooth-fit principle applies as soon as the payoff function is differentiable. A key feature of our proof is that it relies on a localtime argument that remains valid in the case of optimal stopping problems with finite maturity. Moreover, the criterion enables us to present a class of optimal stopping problems associated with a convex payoff function whose value function is not convex. Finally, in Section 5 we give illustrative examples arising from real option models.

\section{The model, notation, and assumptions}

Consider a probability space $\left(\Omega, \mathcal{F},\left(\mathcal{F}_{t}\right)_{t \geq 0}, \mathrm{P}\right)$, where $\left(\mathcal{F}_{t}\right)_{t \geq 0}$ satisfies the usual conditions. We will assume that the state process $\left(X_{t}\right)_{t \geq 0}$ is a solution to the following stochastic differential equation, where $\left(W_{t}\right)_{t \geq 0}$ is a standard $\widetilde{\mathcal{F}}_{t}$-Brownian motion:

$$
\frac{\mathrm{d} X_{t}}{X_{t}}=\left(r-\delta\left(X_{t}\right)\right) \mathrm{d} t+\sigma\left(X_{t}\right) \mathrm{d} W_{t} .
$$

Throughout this paper, we will assume the following hypothesis to hold. 
Assumption 2.1. The function $\delta: \mathbb{R}_{+} \rightarrow \mathbb{R}_{+}$is bounded and continuous and the function $\sigma: \mathbb{R}_{+} \rightarrow \mathbb{R}_{+}$is bounded and continuous with inf $x \geq 0 \sigma(x)>0$. We also assume that $x \sigma(x)$ is Lipschitz continuous.

Under Assumption 2.1, the coefficients satisfy the following local integrability condition:

$$
\text { there exists an } \varepsilon \text { such that } \int_{x-\varepsilon}^{x+\varepsilon} \frac{1+y|r-\delta(y)|}{y^{2} \sigma(y)^{2}} \mathrm{~d} y<\infty \text { for all } x>0 .
$$

This guarantees that the stochastic differential equation (2.1) has a weak solution which is unique in the sense of probability law (see [13, pp. 339-344]). Moreover, the solution $X$ is regular; that is, $\mathrm{P}_{x}\left(T_{y}<\infty\right)>0$ for $x, y>0$, where

$$
T_{y}=\inf \left\{t \geq 0: X_{t}=y\right\} .
$$

Note that, under Assumption 2.1, the boundaries 0 and $\infty$ are natural. However, the stochastic differential equation

$$
\frac{\mathrm{d} \tilde{X}_{t}}{\tilde{X}_{t}}=r \mathrm{~d} t+\sigma\left(\tilde{X}_{t}\right) \mathrm{d} W_{t}
$$

admits a strong solution that satisfies $\mathrm{E}\left(\sup _{0 \leq t \leq T}\left|\tilde{X}_{t}\right|^{p}\right)<\infty$ for all positive numbers $p$ and $T$. Therefore, a comparison result (see [13, Proposition 2.18]) gives $\mathrm{E}\left(\sup _{0 \leq t \leq T}\left|X_{t}\right|^{p}\right)<\infty$ for all positive numbers $p$ and $T$.

Consider an investment project with payoff function $g$ mapping $\mathbb{R}_{+}$into $\mathbb{R}$ and define the associated value function

$$
V(x)=\sup _{\tau \in \mathcal{T}_{0, \infty}} \mathrm{E}\left(\mathrm{e}^{-r \tau} g\left(X_{\tau}^{x}\right)\right),
$$

where $\mathcal{T}_{0, \infty}$ is the set of all $\left(\mathcal{F}_{t}\right)_{t \geq 0}$-adapted stopping times with values in $[0, \infty]$. Let us recall the basics of optimal stopping theory (we refer the reader to [8] and [14, Theorems D9 and D12]; see also [20, Theorems 10.1.9 and 10.1.12]). Introduce the set

$$
E=\{x>0: V(x)=g(x)\}
$$

and assume that $g$ is continuous. Then the stopping time $\tau_{E}$ is optimal under the integrability condition $\sup _{t>0} \mathrm{e}^{-r t} g\left(X_{t}\right) \in L^{1}(\Omega, \mathcal{F}, \mathrm{P})$. Moreover, the process $\left(\mathrm{e}^{-r\left(t \wedge \tau_{E}\right)} V\left(X_{t \wedge \tau_{E}}\right)\right)_{t \geq 0}$ is a martingale. Therefore, if $E$ is empty then there is no optimal stopping time and the process $\left(\mathrm{e}^{-r t} V\left(X_{t}\right)\right)_{t \geq 0}$ is a martingale. Note that, under the integrability condition, the value function is finite for every $x>0$.

If the integrability condition fails, it may happen that the stopping set is nonempty but the stopping time $\tau_{E}$ fails to be optimal (see [3, Example 6.5]). Dayanik and Karatzas [3] gave conditions sufficient to ensure both that the value function is finite and that the stopping time $\tau_{E}$ is optimal. To this end, they introduced the second-order differential operator $\mathcal{L}$ such that

$$
\mathcal{L} u=\frac{1}{2} x^{2} \sigma(x)^{2} u^{\prime \prime}+x(r-\delta(x)) u^{\prime} .
$$

Suppose that Assumption 2.1 holds. Then the differential equation $\mathcal{L} u=r u$ has two linearly independent positive solutions, say $\varphi$ and $\psi$, which are uniquely determined (up to multiplication by a constant) if we require one of them, say $\psi$, to be strictly increasing and the other to be strictly decreasing (see [2, Chapter 2]). Finally, under Assumption 2.1, Dayanik and Karatzas proved the following three results (see [3, Propositions 5.10, 5.13, and 5.14]). 
Proposition 2.1. The value function $V$ is finite for every $x>0$ if and only if

$$
l_{0}:=\limsup _{x \rightarrow 0} \frac{g(x)}{\varphi(x)} \quad \text { and } \quad l_{\infty}:=\limsup _{x \rightarrow \infty} \frac{g(x)}{\psi(x)}
$$

are both finite.

Proposition 2.2. If $g$ is continuous and $l_{0}=l_{\infty}=0$, then $\tau_{E}$ is an optimal stopping time.

Proposition 2.3. Suppose that $l_{0}$ and $l_{\infty}$ are finite, that one of them is strictly positive, and that $g$ is continuous. Then $\tau_{E}$ is an optimal stopping time if and only if

$$
\text { there exists no } r>0 \text { such that }(0, r) \subset C \text { for } l_{0}>0
$$

and

$$
\text { there exists no } l>0 \text { such that }(l, \infty) \subset C \text { for } l_{0}>0 \text {. }
$$

Note that, according to Proposition 2.3, optimality of the threshold strategy implies optimality of the stopping time $\tau_{E}$ for any nondecreasing convex payoff function. In this paper we do not assume the integrability condition to hold and we instead work on sufficient conditions relying on the signed measure

$$
\mathcal{A} g:=\mathcal{L} g-r g .
$$

To be meaningful, we will restrict the class of payoff functions by making the following assumption.

Assumption 2.2. (i) There exists an $x_{0}$ such that $g\left(x_{0}\right)>0$.

(ii) Let $D=\left\{a_{1}, \ldots, a_{n}\right\}$, where $n \in \mathbb{N}$ and the $a_{i}$ are positive real numbers such that $a_{1}<a_{2}<\cdots<a_{n}$. Suppose that $g$ is a continuous function on $\mathbb{R}^{+}$such that $g^{\prime}$ and $g^{\prime \prime}$ exist and are continuous on $\mathbb{R}^{+} \backslash D$ and the limits

$$
g^{\prime}\left(a_{i}^{ \pm}\right):=\lim _{x \rightarrow a_{i}^{ \pm}} g^{\prime}(x) \text { and } g^{\prime \prime}\left(a_{i}^{ \pm}\right):=\lim _{x \rightarrow a_{i}^{ \pm}} g^{\prime \prime}(x)
$$

exist and are finite.

(iii) There exist $C>0$ and $p>0$ such that, for every $x>a_{n}$,

$$
|g(x)|+\left|g^{\prime}(x)\right|+\left|g^{\prime \prime}(x)\right| \leq C\left(1+|x|^{p}\right) .
$$

Remark 2.1. Let $T>0$. Then a standard application of the Burkholder-Davis-Gundy inequality shows that the process $\left(\int_{0}^{t} \mathrm{e}^{-r s} g^{\prime}\left(X_{S}\right) \sigma\left(X_{s}\right) X_{s} \mathrm{~d} W_{s}\right)_{0 \leq t \leq T}$ is a square-integrable martingale for any function $g$ satisfying Assumption 2.2.

Remark 2.2. Under Assumption 2.2, the signed measure $\mathcal{A} g$ must be decomposed as

$$
\mathcal{A} g(\mathrm{~d} x)=A g(x) \mathrm{d} x+\sum_{i=1}^{n} \frac{\sigma\left(a_{i}\right)^{2} a_{i}^{2}}{2}\left(g^{\prime}\left(a_{i}^{+}\right)-g^{\prime}\left(a_{i}^{-}\right)\right) \delta_{a_{i}},
$$

where $\delta_{a_{i}}$ stands for the Dirac measure at the level $a_{i}$ and $A g(x)=(\mathscr{L} g(x)-r g(x)) \mathbf{1}_{\{x \notin D\}}$. 


\section{Nonemptiness of the exercise region}

In this section we study conditions sufficient to ensure the nonemptiness of the exercise region. We recall that, for optimal stopping problems with finite maturities, Villeneuve [22, Theorem 2.1] gave the following characterization: the exercise region is empty if and only if $\mathrm{A} g$ is a nonzero, positive measure.

Unfortunately, this characterization fails in the case of perpetual-maturity optimal stopping problems, as illustrated in the following example.

Example 3.1. Let us consider the payoff function

$$
g(x)=\min (x-1,(1-\varepsilon) x-(1-2 \varepsilon)) \quad \text { with } 0<\varepsilon<\frac{1}{2}
$$

and the associated value function

$$
V(x)=\sup _{\tau \in \mathcal{T}_{0, \infty}} \mathrm{E}\left(\mathrm{e}^{-r \tau} g\left(X_{\tau}^{x}\right)\right)
$$

where

$$
X_{t}^{x}=x \mathrm{e}^{\left(r-\sigma^{2} / 2\right) t+\sigma W_{t}} .
$$

The process $\left(\mathrm{e}^{-r t} X_{t}\right)_{0 \leq t<\infty}$ is a positive martingale. Therefore, [13, Problem 1.3.16] and the optional sampling theorem give $\mathrm{E}\left(\mathrm{e}^{-r \tau} X_{\tau}\right) \leq x$ for every $\tau \in \mathcal{J}_{0, \infty}$, whence $V(x) \leq(1-\varepsilon) x$. On the other hand, for all $t \geq 0$ we have

$$
\begin{aligned}
V(x) & \geq \mathrm{E}\left(\mathrm{e}^{-r t} g\left(X_{t}^{x}\right)\right) \\
& =\mathrm{E}\left(\mathrm{e}^{-r t}\left((1-\varepsilon) X_{t}^{x}-(1-2 \varepsilon)\right)\right)+\varepsilon \mathrm{E}\left(\mathrm{e}^{-r t}\left(X_{t}^{x}-2\right) \mathbf{1}_{\left\{X_{t}^{x} \leq 2\right\}}\right) \\
& \geq(1-\varepsilon) x-(1-2 \varepsilon) \mathrm{e}^{-r t}-2 \varepsilon \mathrm{E}\left(\mathrm{e}^{-r t} \mathbf{1}_{\left\{X_{t}^{x} \leq 2\right\}}\right) .
\end{aligned}
$$

Letting $t$ tend to $\infty$, we obtain $V(x) \geq(1-\varepsilon) x$.

Thus, we have $V(x)=(1-\varepsilon) x$, and the exercise region is consequently empty. However,

$$
\mathcal{A} g=r \mathbf{1}_{\{x<2\}}+r(1-2 \varepsilon) \mathbf{1}_{\{x>2\}}-2 \varepsilon \sigma^{2} \delta_{2}
$$

(where, recall, $\delta_{a}$ stands for the Dirac measure at the level $a$ ) is not a positive measure.

Remark 3.1. The statement of sufficiency in the characterization result of Villeneuve [22, Theorem 2.1] remains true for perpetual-maturity optimal stopping problems; that is, if $\mathcal{A g}$ is a positive measure then the exercise region is empty.

The next proposition will allow us to work with positive payoff functions in what follows. Let us define $g^{+}=\max (g, 0)$ and $g^{-}=(-g)^{+}$.

Proposition 3.1. Suppose that Assumption 2.1 holds. Let $g$ be a function satisfying Assumption 2.2 and such that

$$
\lim _{t \rightarrow \infty} \mathrm{E}_{x}\left(\mathrm{e}^{-r t} g^{-}\left(X_{t}\right)\right)=0
$$

Then

$$
V(x)=\sup _{\tau \in \mathcal{T}_{0, \infty}} \mathrm{E}_{x}\left(\mathrm{e}^{-r \tau} g^{+}\left(X_{\tau}\right)\right)
$$


Proof. Let us consider the following optimal stopping problems with finite maturity:

$$
V(t, x)=\sup _{\tau \in \widetilde{\mathcal{T}}_{0, t}} \mathrm{E}_{x}\left(\mathrm{e}^{-r \tau} g\left(X_{\tau}\right)\right), \quad V_{+}(t, x)=\sup _{\tau \in \widetilde{\mathcal{T}}_{0, t}} \mathrm{E}_{x}\left(\mathrm{e}^{-r \tau} g^{+}\left(X_{\tau}\right)\right) .
$$

Moreover, let us introduce the optimal stopping time

$$
\tau_{t}^{*}=\inf \left\{s \in[0, t]: V_{+}\left(s, X_{s}\right)=g^{+}\left(X_{s}\right)\right\} .
$$

According to optimal stopping theory, $\tau_{t}^{*}$ is an optimal stopping time and, by condition (i) of Assumption 2.2, we have

$$
V_{+}\left(\tau_{t}^{*}, X_{\tau_{t}^{*}}\right)=g^{+}\left(X_{\tau_{t}^{*}}\right)>0 \quad \text { on }\left\{\tau_{t}^{*}<t\right\}
$$

Therefore,

$$
\begin{aligned}
V_{+}(t, x) & =\mathrm{E}_{x}\left(\mathrm{e}^{-r \tau_{t}^{*}} g^{+}\left(X_{\tau_{t}^{*}}\right) \mathbf{1}_{\left\{\tau_{t}^{*}<t\right\}}\right)+\mathrm{E}_{x}\left(\mathrm{e}^{-r t} g^{+}\left(X_{t}\right) \mathbf{1}_{\left\{\tau_{t}^{*}=t\right\}}\right) \\
& =\mathrm{E}_{x}\left(\mathrm{e}^{-r \tau_{t}^{*}} g\left(X_{\tau_{t}^{*}}\right)\right)+\mathrm{E}_{x}\left(\mathrm{e}^{-r t} g^{-}\left(X_{t}\right) \mathbf{1}_{\left\{\tau_{t}^{*}=t\right\}}\right) \\
& \leq V(t, x)+\mathrm{E}_{x}\left(\mathrm{e}^{-r t} g^{-}\left(X_{t}\right) \mathbf{1}_{\left\{\tau_{t}^{*}=t\right\}}\right) .
\end{aligned}
$$

From (3.1) we obtain

$$
\sup _{\tau \in \mathcal{T}_{0, \infty}} \mathrm{E}_{x}\left(\mathrm{e}^{-r \tau} g^{+}\left(X_{\tau}\right)\right) \leq V(x)
$$

by letting $t$ tend to $\infty$. Since the reverse inequality is obvious, we have the desired result.

Remark 3.2. Proposition 3.1 is valid if $g$ is bounded below, that is, there exists an $M>0$ such that $g(x)>-M$ for every $x>0$.

The next theorem gives a characterization of the nonemptiness of the exercise region for perpetual-maturity optimal stopping problems.

Theorem 3.1. Assume that $g$ is a positive function satisfying Assumption 2.2 and such that

$$
g\left(0^{+}\right)<\infty \text { and } \lim _{x \rightarrow \infty} \frac{g(x)}{\psi(x)}=l_{\infty} \in(0, \infty) .
$$

Then the exercise region $E$ is empty if and only if, for every $x>0$, we have

$$
\liminf _{t \rightarrow \infty} \mathrm{E}_{x}\left(\mathrm{e}^{-r t} g\left(X_{t}\right)\right)>g(x) .
$$

Moreover, if $E$ is empty then $V(x)=\lim _{t \rightarrow \infty} \mathrm{E}_{x}\left(\mathrm{e}^{-r t} g\left(X_{t}\right)\right)$.

Proof. To begin, let us note the following points. According to [3, Propositions 5.10 and 5.13], the value function $V$ is a continuous function satisfying $V(x)<\infty$ for every $x>0$, and since the boundaries 0 and $\infty$ are natural we have (see [10, pp. 128-135])

$$
\psi\left(0^{+}\right)=\lim _{x \rightarrow \infty} \varphi(x)=0 \text { and } \varphi\left(0^{+}\right)=\lim _{x \rightarrow \infty} \psi(x)=\infty .
$$

We shall first prove the sufficient condition for the emptiness of the exercise region $E$. Introduce the notation $T_{t} g(x):=\mathrm{E}_{x}\left(\mathrm{e}^{-r t} g\left(X_{t}\right)\right)$. If $\liminf _{t \rightarrow \infty} T_{t} g(x)>g(x)$ for any $x>0$, then there exists some $t_{0}(x)$ such that $T_{t} g(x)>g(x)$ for $t \geq t_{0}$, which proves the sufficient condition. 
On the other hand, if $E$ is empty then the assumptions on $g$ combined with the results of [3, Proposition 5.4] imply that $V(x)=l_{\infty} \psi(x)$. By definition, $V(x) \geq T_{t} g(x)$ for every $t \geq 0$ and, thus,

$$
\limsup _{t \rightarrow \infty} \mathrm{E}_{x}\left(\mathrm{e}^{-r t} g\left(X_{t}\right)\right) \leq V(x) .
$$

Now fix an $\varepsilon>0$. There exists some $N$ such that, for every $x \geq N, g(x) \geq\left(l_{\infty}-\varepsilon\right) \psi(x)$. Hence,

$$
\begin{aligned}
\mathrm{E}_{x}\left(\mathrm{e}^{-r t} g\left(X_{t}\right)\right) & =\mathrm{E}_{x}\left(\mathrm{e}^{-r t}\left(g\left(X_{t}\right) \mathbf{1}_{\left\{X_{t} \leq N\right\}}+g\left(X_{t}\right) \mathbf{1}_{\left\{X_{t} \geq N\right\}}\right)\right) \\
& \geq \mathrm{E}_{x}\left(\mathrm{e}^{-r t}\left(g\left(X_{t}\right) \mathbf{1}_{\left\{X_{t} \leq N\right\}}+\left(l_{\infty}-\varepsilon\right) \psi\left(X_{t}\right) \mathbf{1}_{\left\{X_{t} \geq N\right\}}\right)\right) \\
& =\left(l_{\infty}-\varepsilon\right) \psi(x)+\mathrm{E}_{x}\left(\mathrm{e}^{-r t} \theta\left(X_{t}\right) \mathbf{1}_{\left\{X_{t} \leq N\right\}}\right),
\end{aligned}
$$

where $\theta(x)=g(x)-\left(l_{\infty}-\varepsilon\right) \psi(x)$ is continuous on $[0, N]$ since $g\left(0^{+}\right)<\infty$. Therefore,

$$
\mathrm{E}_{x}\left(\mathrm{e}^{-r t}\left|\theta\left(X_{t}\right)\right| \mathbf{1}_{\left\{X_{t} \leq N\right\}}\right) \leq M_{N} \mathrm{e}^{-r t},
$$

where

$$
M_{N}=\sup _{x \in[0, N]}|\theta(x)| .
$$

Letting $t$ tend to $\infty$, and recalling that $V(x)=l_{\infty} \psi(x)$, we obtain

$$
\liminf _{t \rightarrow \infty} \mathrm{E}_{x}\left(\mathrm{e}^{-r t} g\left(X_{t}\right)\right) \geq V(x)
$$

which implies the result.

Remark 3.3. The set $E$ is nonempty for any bounded function $g$ satisfying Assumption 2.2. If $E$ were empty for such a function, then $V$ would be identically 0 by Theorem 3.1, which is incompatible with condition (i) of the assumption.

The next result gives another characterization if we assume in addition that

$$
\mathrm{E}_{x}\left(\int_{0}^{\infty} \mathrm{e}^{-r s}\left|A g\left(X_{s}\right)\right| \mathrm{d} s\right)<\infty
$$

By $L_{s}^{a_{i}}$ we denote the local time of $X$ at the level $a_{i}$.

Corollary 3.1. Under hypothesis (3.3), we have $E=\varnothing$ if and only if

$$
\mathrm{E}_{x}\left(\int_{0}^{\infty} \mathrm{e}^{-r s} A g\left(X_{s}\right) \mathrm{d} s\right)+\sum_{i=1}^{N} \frac{\sigma\left(a_{i}\right)^{2} a_{i}^{2}}{2}\left(g^{\prime}\left(a_{i}^{+}\right)-g^{\prime}\left(a_{i}^{-}\right)\right) \mathrm{E}_{x}\left(\int_{0}^{\infty} \mathrm{e}^{-r s} \mathrm{~d} L_{s}^{a_{i}}\right)>0
$$

for all $x>0$.

Proof. The Itô-Tanaka formula [13, Theorem 7.1] and Remark 2.1 imply that, for every $t \geq 0$ and any function $h$ satisfying Assumption 2.2,

$$
\begin{aligned}
\mathrm{E}_{x}\left(\mathrm{e}^{-r t} h\left(X_{t}\right)\right)= & h(x)+\mathrm{E}_{x}\left(\int_{0}^{t} \mathrm{e}^{-r s} A h\left(X_{s}\right) \mathrm{d} s\right) \\
& +\sum_{i=1}^{n} \frac{\sigma\left(a_{i}\right)^{2} a_{i}^{2}}{2}\left(h^{\prime}\left(a_{i}^{+}\right)-h^{\prime}\left(a_{i}^{-}\right)\right) \mathrm{E}_{x}\left(\int_{0}^{t} \mathrm{e}^{-r s} \mathrm{~d} L_{s}^{a_{i}}\right) .
\end{aligned}
$$


Applying the previous equality to $h(x)=(a-x)_{+}$, we obtain

$$
\begin{aligned}
\frac{\sigma(a)^{2} a^{2}}{2} \mathrm{E}_{x}\left(\int_{0}^{t} \mathrm{e}^{-r s} \mathrm{~d} L_{s}^{a}\right)= & \mathrm{E}_{x}\left(\mathrm{e}^{-r t}\left(a-X_{t}\right)_{+}\right)-(a-x)_{+} \\
& +\mathrm{E}_{x}\left(\int_{0}^{t} \mathrm{e}^{-r s}\left(r a-\delta\left(X_{s}\right) X_{s}\right) \mathbf{1}_{\left\{X_{s}<a\right\}} \mathrm{d} s\right) \\
\leq & a+a\left(1-\mathrm{e}^{-r t}\right) \\
\leq & 2 a .
\end{aligned}
$$

Therefore, the monotone convergence theorem yields

$$
\mathrm{E}_{x}\left(\int_{0}^{\infty} \mathrm{e}^{-r s} \mathrm{~d} L_{s}^{a}\right)<\infty .
$$

Now, hypothesis (3.3) gives

$$
\begin{aligned}
\lim _{t \rightarrow \infty} \mathrm{E}_{x}\left(\mathrm{e}^{-r t} g\left(X_{t}\right)\right)= & g(x)+\mathrm{E}_{x}\left(\int_{0}^{\infty} \mathrm{e}^{-r s} A g\left(X_{s}\right) \mathrm{d} s\right) \\
& +\sum_{i=1}^{n} \frac{\sigma\left(a_{i}\right)^{2} a_{i}^{2}}{2}\left(g^{\prime}\left(a_{i}^{+}\right)-g^{\prime}\left(a_{i}^{-}\right)\right) \mathrm{E}_{x}\left(\int_{0}^{\infty} \mathrm{e}^{-r s} \mathrm{~d} L_{s}^{a_{i}}\right),
\end{aligned}
$$

which yields the conclusion.

We shall prove below that a necessary condition for the emptiness of the exercise region under the assumptions of Theorem 3.1 is that $g\left(0^{+}\right) \leq 0$. Note also that [9, Theorem 8.1] asserts that the exercise region associated with a convex payoff function with $g(0)=0$ is empty if $\delta=0$. The next two lemmas (which are probably well known) complete the study. We give their proofs for the sake of completeness.

Lemma 3.1. Assume that $g$ is a positive function satisfying the assumptions of Theorem 3.1 with $g(0)>0$. Then the exercise region is nonempty.

Proof. We will provide a proof by contradiction, by assuming that $E$ is empty. Thus, the process $\left(\mathrm{e}^{-r t} V\left(X_{t}\right)\right)_{0 \leq t<\infty}$ is a martingale and $V$ can therefore be written as the linear combination $V(x)=A \psi(x)+B \varphi(x) \geq g(x)$ for every $x>0$, where $A$ and $B$ are real constants. Since $g\left(0^{+}\right)<\infty$, we have $\lim \sup _{x \rightarrow 0} g(x) / \varphi(x)=0$. Therefore, [3, Proposition 5.10] yields $\lim \sup _{x \rightarrow 0} V(x) / \varphi(x)=0$ and, thus, $B=0$ using (3.2). However, $\lim _{x \rightarrow 0} A \psi(x)=0$, yielding $g\left(0^{+}\right)=0$, which is a contradiction.

Lemma 3.2. Let $g$ be a nondecreasing, concave function satisfying Assumption 2.2 with $g(0)=0$. Then $V(x)=g(x)$.

Proof. The assumptions on $g$ imply that $x g^{\prime}(x)-g(x)$ is nonpositive at every $x$ where $g$ is differentiable. Since $g$ satisfies Assumption 2.2, $\mathcal{A} g(\mathrm{~d} x)$ is a nonpositive measure. Therefore, the Itô-Tanaka formula implies that the process $\left(\mathrm{e}^{-r t} g\left(X_{t}\right)\right)_{t \geq 0}$ is a supermartingale, which yields the result. 


\section{Main results}

\subsection{Optimal threshold strategies}

In this section we give an easily testable condition sufficient to ensure that threshold strategies are optimal for the payoff functions satisfying Assumption 2.2. We start with a lemma that gives a sufficient condition for the points of the set $D$ to be in the continuation region.

Lemma 4.1. Let $g$ be a positive function satisfying Assumption 2.2 and let $a \in D$ be such that $g^{\prime}\left(a^{+}\right)>g^{\prime}\left(a^{-}\right)$. Then $V(a)>g(a)$.

Proof. Taylor's formula gives

$$
g(x)=g(a)+(x-a)_{+} g^{\prime}\left(a^{+}\right)-(x-a)_{-} g^{\prime}\left(a^{-}\right)+(x-a) \varepsilon(x-a),
$$

where $\lim _{y \rightarrow 0} \varepsilon(y)=0$. Hence, the Itô-Tanaka formula yields

$$
\begin{aligned}
g\left(X_{t}\right)= & g(a)+\left(g^{\prime}\left(a^{+}\right)-g^{\prime}\left(a^{-}\right)\right) \frac{\sigma(a)^{2} a^{2}}{2} L_{t}^{a} \\
& +\int_{0}^{t}\left(g^{\prime}\left(a^{+}\right) \mathbf{1}_{\left\{X_{s}>a\right\}}-g^{\prime}\left(a^{-}\right) \mathbf{1}_{\left\{X_{s}<a\right\}}\right) \mathrm{d} X_{s}+\left(X_{t}-a\right) \varepsilon\left(X_{t}-a\right) .
\end{aligned}
$$

Thus,

$$
\begin{gathered}
\left(X_{t}-a\right)^{2} \varepsilon^{2}\left(X_{t}-a\right) \leq 4\left(g^{2}\left(X_{t}\right)+g^{2}(a)+\left(g^{\prime}\left(a^{+}\right)-g^{\prime}\left(a^{-}\right)\right)^{2}\left(\frac{\sigma(a)^{2} a^{2}}{2}\right)^{2}\left(L_{t}^{a}\right)^{2}\right. \\
\left.+\left(\int_{0}^{t}\left(g^{\prime}\left(a^{+}\right) \mathbf{1}_{\left\{X_{s}>a\right\}}-g^{\prime}\left(a^{-}\right) \mathbf{1}_{\left\{X_{s}<a\right\}}\right) \mathrm{d} X_{s}\right)^{2}\right),
\end{gathered}
$$

and the Burkholder-Davis-Gundy inequality and condition (iii) of Assumption 2.2 imply that $\left(X_{t}-a\right) \varepsilon\left(X_{t}-a\right)$ is a square-integrable random variable for any $t \geq 0$. Taking expectations, we obtain

$$
\begin{aligned}
\mathrm{E}_{a}\left(g\left(X_{t}\right)\right)= & g(a)+\left(g^{\prime}\left(a^{+}\right)-g^{\prime}\left(a^{-}\right)\right) \frac{\sigma(a)^{2} a^{2}}{2} \mathrm{E}_{a}\left(L_{t}^{a}\right) \\
& +\mathrm{E}_{a}\left(\int_{0}^{t}\left(g^{\prime}\left(a^{+}\right) \mathbf{1}_{\left\{X_{s}>a\right\}}-g^{\prime}\left(a^{-}\right) \mathbf{1}_{\left\{X_{s}<a\right\}}\right)\left(r-\delta\left(X_{s}\right)\right) X_{s} \mathrm{~d} s\right) \\
& +\mathrm{E}_{a}\left(\left(X_{t}-a\right) \varepsilon\left(X_{t}-a\right)\right) .
\end{aligned}
$$

We shall treat each term on the right-hand side of this equation separately. For the first term, the Itô-Tanaka formula and the Burkholder-Davis-Gundy inequality give

$$
\begin{aligned}
\frac{\sigma(a)^{2} a^{2}}{2} \mathrm{E}_{a}\left(L_{t}^{a}\right) & =\mathrm{E}_{a}\left(\left|X_{t}-a\right|\right)-\mathrm{E}_{a}\left(\int_{0}^{t}\left(\mathbf{1}_{\left\{X_{s}>a\right\}}-\mathbf{1}_{\left\{X_{s}<a\right\}}\right)\left(r-\delta\left(X_{s}\right)\right) X_{s} \mathrm{~d} s\right) \\
& \geq \mathrm{E}_{a}\left(\left|\int_{0}^{t} \sigma\left(X_{s}\right) X_{s} \mathrm{~d} W_{s}\right|\right)-\mathrm{E}_{a}\left(\left|\int_{0}^{t}\left(r-\delta\left(X_{s}\right)\right) X_{s} \mathrm{~d} s\right|\right)+o(\sqrt{t}) \\
& \geq c \mathrm{E}_{a}\left(\sqrt{\int_{0}^{t} \sigma^{2}\left(X_{s}\right) X_{s}^{2} \mathrm{~d} s}\right)+o(\sqrt{t}) \\
& =c \sigma(a)^{2} a^{2} \sqrt{t}+o(\sqrt{t}) .
\end{aligned}
$$


On the other hand, writing $b(x)=(r-\delta(x)) x$, we have

$$
\begin{aligned}
\left|\frac{1}{\sqrt{t}} \mathrm{E}_{a}\left(\int_{0}^{t} b\left(X_{s}\right) \mathbf{1}_{\left\{X_{s}>a\right\}} \mathrm{d} s\right)\right| \leq & \frac{1}{\sqrt{t}}|b(a)| \int_{0}^{t} \mathrm{P}\left(X_{s}>a\right) \mathrm{d} s \\
& +\frac{1}{\sqrt{t}} \mathrm{E}_{a}\left(\int_{0}^{t}\left|b\left(X_{s}\right)-b(a)\right| \mathrm{d} s\right) \\
\leq & |b(a)| \sqrt{t}+o(\sqrt{t}),
\end{aligned}
$$

which allows us to conclude that the second term is $o(\sqrt{t})$.

To study the third term, we fix an $\eta>0$ such that $|\varepsilon(y)| \leq \eta$ for $|y| \leq h$. Hence,

$$
\begin{aligned}
\mathrm{E}_{a}\left(\left(X_{t}-a\right) \varepsilon\left(X_{t}-a\right)\right)= & \mathrm{E}_{a}\left(\left(X_{t}-a\right) \varepsilon\left(X_{t}-a\right) \mathbf{1}_{\left\{\left|X_{t}-a\right| \leq h\right\}}\right) \\
& +\mathrm{E}_{a}\left(\left(X_{t}-a\right) \varepsilon\left(X_{t}-a\right) \mathbf{1}_{\left\{\left|X_{t}-a\right| \geq h\right\}}\right) \\
\leq & \eta \mathrm{E}_{a}\left(\left|X_{t}-a\right|\right)+\left[\mathrm{E}_{a}\left(\left(X_{t}-a\right)^{2} \varepsilon^{2}\left(X_{t}-a\right)\right) \mathrm{P}\left(\left|X_{t}-a\right| \geq h\right)\right]^{1 / 2} \\
\leq & C \eta \sqrt{t}+o(\sqrt{t}),
\end{aligned}
$$

where we have used the fact that $\mathrm{P}\left(\left|X_{t}-a\right| \geq h\right)=o(t)$ (see, for instance, [6, Proposition 2.1]). Therefore,

$$
\lim _{t \rightarrow 0} \frac{\mathrm{E}_{a}\left(\left(X_{t}-a\right) \varepsilon\left(X_{t}-a\right)\right)}{\sqrt{t}}=0 .
$$

Finally,

$$
\begin{aligned}
\mathrm{E}_{a}\left(\mathrm{e}^{-r t} g\left(X_{t}\right)\right) & =\mathrm{E}_{a}\left(g\left(X_{t}\right)\right)+o(t) \\
& \geq g(a)+\left(g^{\prime}\left(a^{+}\right)-g^{\prime}\left(a^{-}\right)\right) c_{1} \sigma(a)^{2} a^{2} \sqrt{t}+o(\sqrt{t}) .
\end{aligned}
$$

Consequently, for small enough $t$ we obtain

$$
\mathrm{E}_{a}\left(\mathrm{e}^{-r t} g\left(X_{t}\right)\right)>g(a),
$$

which implies the result.

Remark 4.1. It should be pointed out that the previous result remains valid for optimal stopping problems with finite maturities. That is, for every $T>0, V_{T}(a)>g(a)$ for $a \in D$ such that $g^{\prime}\left(a^{+}\right)>g^{\prime}\left(a^{-}\right)$.

Lemma 4.2. Assume that $x_{0} \in E$ and that $g$ is a payoff function satisfying Assumption 2.2. If $\mathcal{A} g$ is a nonpositive measure on the open interval $\left(x_{0}, \infty\right)$, then $E$ contains the interval $\left[x_{0}, \infty\right)$.

Proof. As a first step, we shall prove the equality

$$
V(x)=\sup _{\tau \in \mathcal{T}_{0, \infty}} \mathrm{E}\left(\mathrm{e}^{-r\left(\tau \wedge \tau_{E}\right)} g\left(X_{\tau \wedge \tau_{E}}^{x}\right)\right) .
$$

Let us consider a stopping time $\tau$. The strong Markov property (see [13, Theorem 5.4.20]) implies that

$$
\begin{aligned}
\mathrm{E}_{x}\left(\mathrm{e}^{-r \tau} g\left(X_{\tau}\right) \mathbf{1}_{\left\{\tau>\tau_{E}\right\}} \mathbf{1}_{\left\{\tau_{E}<\infty\right\}}\right) & =\mathrm{E}_{x}\left(\mathrm{E}\left(\mathrm{e}^{-r \tau} g\left(X_{\tau}\right) \mid \mathcal{F}_{\tau_{E}}\right) \mathbf{1}_{\left\{\tau>\tau_{E}\right\}} \mathbf{1}_{\left\{\tau_{E}<\infty\right\}}\right) \\
& \leq \mathrm{E}_{x}\left(\mathrm{e}^{-r \tau_{E}} V\left(X_{\tau_{E}}\right) \mathbf{1}_{\left\{\tau>\tau_{E}\right\}} \mathbf{1}_{\left\{\tau_{E}<\infty\right\}}\right) \\
& =\mathrm{E}_{x}\left(\mathrm{e}^{-r \tau_{E}} g\left(X_{\tau_{E}}\right) \mathbf{1}_{\left\{\tau>\tau_{E}\right\}} \mathbf{1}_{\left\{\tau_{E}<\infty\right\}}\right)
\end{aligned}
$$


where the last equality follows from the definition of the stopping region. Hence,

$$
\begin{aligned}
\mathrm{E}_{x}\left(\mathrm{e}^{-r \tau} g\left(X_{\tau}\right)\right) & =\mathrm{E}_{x}\left(\mathrm{e}^{-r \tau} g\left(X_{\tau}\right) \mathbf{1}_{\left\{\tau \leq \tau_{E}\right\}}\right)+\mathrm{E}_{x}\left(\mathrm{e}^{-r \tau} g\left(X_{\tau}\right) \mathbf{1}_{\left\{\tau>\tau_{E}\right\}} \mathbf{1}_{\left\{\tau_{E}<\infty\right\}}\right) \\
& \leq \mathrm{E}_{x}\left(\mathrm{e}^{-r \tau} g\left(X_{\tau}\right) \mathbf{1}_{\left\{\tau \leq \tau_{E}\right\}}\right)+\mathrm{E}_{x}\left(\mathrm{e}^{-r \tau_{E}} g\left(X_{\tau_{E}}\right) \mathbf{1}_{\left\{\tau>\tau_{E}\right\}} \mathbf{1}_{\left\{\tau_{E}<\infty\right\}}\right) \\
& =\mathrm{E}_{x}\left(\mathrm{e}^{-r\left(\tau \wedge \tau_{E}\right)} g\left(X_{\tau \wedge \tau_{E}}\right)\right) .
\end{aligned}
$$

Therefore,

$$
V(x) \leq \sup _{\tau \in \mathcal{T}_{0, \infty}} \mathrm{E}\left(\mathrm{e}^{-r\left(\tau \wedge \tau_{E}\right)} g\left(X_{\tau \wedge \tau_{E}}^{x}\right)\right)
$$

Since the reverse inequality is obvious, we have proved the first step.

The Itô-Tanaka formula yields

$$
\begin{aligned}
\mathrm{E}_{x}\left(\mathrm{e}^{-r\left(\tau \wedge \tau_{E}\right)} g\left(X_{\tau \wedge \tau_{E}}\right)\right)= & g(x)+\mathrm{E}\left(\int_{0}^{\tau \wedge \tau_{E}} \mathrm{e}^{-r s} A g\left(X_{s}\right) \mathrm{d} s\right) \\
& +\sum_{i=1}^{n} \frac{\sigma^{2}\left(a_{i}\right) a_{i}^{2}}{2}\left(g^{\prime}\left(a_{i}^{+}\right)-g^{\prime}\left(a_{i}^{-}\right)\right) \mathrm{E}_{x}\left(\int_{0}^{\tau \wedge \tau_{E}} \mathrm{e}^{-r s} \mathrm{~d} L_{s}^{a_{i}}\right),
\end{aligned}
$$

with $A g(x)=\frac{1}{2} x^{2} \sigma(x)^{2} g^{\prime \prime}(x)+x(r-\delta(x)) g^{\prime}(x)-r g(x)$. The assumption on the measure $\mathcal{A} g$ gives $g^{\prime}\left(a_{i}^{+}\right)-g^{\prime}\left(a_{i}^{-}\right) \leq 0$ for every $a_{i} \geq x_{0}$. Moreover, let us note that, for $x \geq x_{0}, X_{s}^{x} \geq x_{0}$ on the random interval $\left[0, \tau \wedge \tau_{E}\right]$. Thus,

$$
\begin{aligned}
\mathrm{E}_{x}\left(\mathrm{e}^{-r\left(\tau \wedge \tau_{E}\right)} g\left(X_{\tau \wedge \tau_{E}}\right)\right)= & g(x)+\mathrm{E}\left(\int_{0}^{\tau \wedge \tau_{E}} \mathrm{e}^{-r s} A g\left(X_{S}\right) \mathrm{d} s\right) \\
& +\sum_{a_{i} \geq x_{0}} \frac{\sigma^{2}\left(a_{i}\right) a_{i}^{2}}{2}\left(g^{\prime}\left(a_{i}^{+}\right)-g^{\prime}\left(a_{i}^{-}\right)\right) \mathrm{E}_{x}\left(\int_{0}^{\tau \wedge \tau_{E}} \mathrm{e}^{-r s} \mathrm{~d} L_{s}^{a_{i}}\right) \\
\leq & g(x) .
\end{aligned}
$$

From the first step, we thus conclude that $x \in E$.

We are now in a position to give a criterion for optimality of threshold strategies for perpetualmaturity optimal stopping problems.

Theorem 4.1. Let $g$ be a positive payoff function satisfying Assumption 2.2. Assume that there exists an $x_{1}$ such that $\mathcal{A} g$ is a nonzero, positive measure on $\left(0, x_{1}\right)$ and a nonpositive measure on $\left(x_{1}, \infty\right)$ with

$$
\lim _{x \rightarrow \infty} A g(x)=-l<0 \quad \text { and } \quad \mathrm{E}_{x}\left(\int_{0}^{\infty} \mathrm{e}^{-r s}\left|A g\left(X_{S}\right)\right| \mathrm{d} s\right)<\infty .
$$

Then $E=\left[x^{*}, \infty\right)$ with $x^{*} \geq x_{1}$, and the hitting time $T_{x^{*}}$ is optimal.

Proof. First we shall prove that $E$ is nonempty. Let us define

$$
\begin{aligned}
R(x)= & \mathrm{E}_{x}\left(\int_{0}^{\infty} \mathrm{e}^{-r s} \operatorname{Ag}\left(X_{s}\right) \mathrm{d} s\right) \\
& +\sum_{i=1}^{n} \frac{\sigma\left(a_{i}\right)^{2} a_{i}^{2}}{2}\left(g^{\prime}\left(a_{i}^{+}\right)-g^{\prime}\left(a_{i}^{-}\right)\right) \mathrm{E}_{x}\left(\int_{0}^{\infty} \mathrm{e}^{-r s} \mathrm{~d} L_{s}^{a_{i}}\right) .
\end{aligned}
$$


For $x>a_{n}$ and $a \in D$, the Itô-Tanaka formula yields

$$
\begin{aligned}
\frac{\sigma(a)^{2} a^{2}}{2} \mathrm{E}_{x}\left(\int_{0}^{t} \mathrm{e}^{-r s} \mathrm{~d} L_{s}^{a}\right)= & \mathrm{E}_{x}\left(\mathrm{e}^{-r t}\left(a-X_{t}\right)\right)_{+} \\
& +\mathrm{E}_{x}\left(\int_{0}^{t} \mathrm{e}^{-r s}\left(r a-\delta\left(X_{S}\right) X_{S}\right) \mathbf{1}_{\left\{X_{s}<a\right\}} \mathrm{d} s\right) \\
\leq & a \mathrm{e}^{-r t}+r a \int_{0}^{t} \mathrm{e}^{-r s} \mathrm{P}_{x}\left(X_{s}<a\right) \mathrm{d} s
\end{aligned}
$$

Therefore,

$$
\frac{\sigma(a)^{2} a^{2}}{2} \mathrm{E}_{x}\left(\int_{0}^{\infty} \mathrm{e}^{-r s} \mathrm{~d} L_{s}^{a}\right) \leq r a \int_{0}^{\infty} \mathrm{e}^{-r s} \mathrm{P}_{x}\left(X_{s}<a\right) \mathrm{d} s,
$$

from which we deduce that

$$
\lim _{x \rightarrow \infty} \mathrm{E}_{x}\left(\int_{0}^{\infty} \mathrm{e}^{-r s} \mathrm{~d} L_{s}^{a}\right)=0,
$$

by dominated convergence. According to [17, p. 32], we have

$$
\lim _{x \rightarrow \infty} \mathrm{E}_{x}\left(\int_{0}^{\infty} \mathrm{e}^{-r s} A g\left(X_{s}\right) \mathrm{d} s\right)=\frac{1}{r} \lim _{x \rightarrow \infty} A g(x)=-\frac{l}{r}<0 ;
$$

thus, $R$ is nonpositive for large enough $x$. Therefore, $E$ is nonempty according to Corollary 3.1.

Now, that $\mathcal{A} g$ is a nonzero, positive measure on $\left(0, x_{1}\right)$ means either

$$
\mathcal{A} g(x)>0 \text { on every open set } U \subset \bar{U} \subset\left(0, x_{1}\right) \backslash D
$$

or

$$
\text { there exists an } a \in D \cup\left(0, x_{1}\right) \text { such that } g^{\prime}\left(a^{+}\right)>g^{\prime}\left(a^{-}\right) \text {. }
$$

In either case, for $x \in\left(0, x_{1}\right)$ we have

$$
\mathrm{E}_{x}\left(\int_{0}^{T_{x_{1}}} \mathrm{e}^{-r s} \mathcal{A} g\left(X_{S}\right) \mathrm{d} s\right)>0 .
$$

Therefore, Dynkin's theorem implies that

$$
\mathrm{E}_{x}\left(\mathrm{e}^{-r T_{x_{1}}} g\left(X_{T_{x_{1}}}\right)\right)>g(x)
$$

for $x \in\left(0, x_{1}\right)$. Now, let $x \in E$. The previous remark implies that $x \geq x_{1}$, so $E$ contains $[x, \infty)$ according to Lemma 4.2. To complete the proof, it suffices to choose $x^{*}=\inf \left\{x \geq x_{1}: x \in E\right\}$. The optimality of $T_{x^{*}}$ follows from Proposition 2.3.

Remark 4.2. Consider an optimal stopping problem with finite maturity $T>0$, and define

$$
V_{t}(x)=\sup _{\tau \in \widetilde{\mathcal{T}}_{0, t}} \mathrm{E}\left(\mathrm{e}^{-r \tau} g\left(X_{\tau}\right)\right)
$$

for $t<T$. Under the assumptions of Theorem 4.1, we have optimality of a threshold strategy, that is,

$$
E_{t}=\left\{x>0: V_{t}(x)=g(x)\right\}=\left[x^{*}(t), \infty\right) .
$$


Remark 4.3. Kotlow [15, Condition 1.1 and Theorem 4] implicitly gave the same sufficient condition for the optimality of a threshold strategy when $g$ is twice continuously differentiable, using partial differential equation techniques.

Example 4.1. Dupuis and Wang [6] gave a sufficient condition based on the stochastic flows method for optimal threshold strategies in the case in which $g(x)=(x-K)_{+}$. Their condition is

$$
\delta(x)+x \delta^{\prime}(x) \geq 0 \text { for every } x>0 .
$$

It should be noted that this condition is not sufficient to ensure the nonemptiness of the exercise region (see the Example 3.1). We prove their result using Theorem 4.1. We have

$$
\mathcal{A} g=\frac{\sigma^{2}}{2} K^{2} \delta_{K}+(r K-x \delta(x)) \mathbf{1}_{\{x>K\}} .
$$

Under Dupuis and Wang's condition, the function $r K-x \delta(x)$ is decreasing and the measure $\mathcal{A} g$ thus satisfies the condition of Theorem 4.1 if, in addition, there exists a real number $x_{0}>K$ such that $x_{0} \delta\left(x_{0}\right)>r K$. For instance, let $\delta(x)=\delta \min (1,1 / x)$ for some positive constant $\delta$, and let $K=1$. For $x>1, \mathcal{A} g(x)=r-\delta$; therefore, $E$ is empty if $r \geq \delta$ and is nonempty and right connected if $r<\delta$.

\subsection{The smooth-fit principle}

In this section we present a new proof of the smooth-fit principle for optimal stopping problems in one space dimension that relies on a local-time argument which does not depend on the maturity. For the perpetual-maturity case, the smooth-fit principle was recently proved by Dayanik and Karatzas [3, Section 7] using their $F$-transformation method.

We start by proving a result in the perpetual-maturity case. This result, which can be viewed as the converse of [1, Theorem 6], shows that, for optimal stopping problems with optimal threshold strategies $\left(E=\left[x^{*}, \infty\right)\right)$, the smooth-fit principle applies and $x^{*}$ satisfies the equation $g^{\prime}(x) \psi(x)-g(x) \psi^{\prime}(x)=0$.

Proposition 4.1. Assume that Assumption 2.1 holds and that $g$ satisfies Assumption 2.2 with $g(0)=0$. If $E=\left[x^{*}, \infty\right)$ and $x^{*} \notin D$, then $V^{\prime}\left(x^{*}\right)=g^{\prime}\left(x^{*}\right)$.

Proof. We will show that the function $g / \psi$ attains a global maximum at $x^{*}$. For $x \leq x^{*}$, we have (see [3, Proposition 5.5]) $V(x)=\left[g\left(x^{*}\right) / \psi\left(x^{*}\right)\right] \psi(x)$. Since $V$ dominates $g$, we have

$$
\frac{g(x)}{\psi(x)} \leq \frac{g\left(x^{*}\right)}{\psi\left(x^{*}\right)} \quad \text { for } x \leq x^{*} .
$$

On the other hand, the process $\left(\mathrm{e}^{-r t} V\left(X_{t}\right)\right)_{t \geq 0}$ is a supermartingale and, thus, for $x \geq x^{*}$,

$$
V\left(x^{*}\right) \geq \mathrm{E}_{x^{*}}\left(\mathrm{e}^{-r T_{x}} V\left(X_{T_{x}}\right)\right)=V(x) \frac{\psi\left(x^{*}\right)}{\psi(x)} .
$$

As the exercise region is the interval $\left[x^{*}, \infty\right)$, we have $V(x)=g(x)$ for $x \geq x^{*}$, that is, $g(x) / \psi(x) \leq g\left(x^{*}\right) / \psi\left(x^{*}\right)$. Now, if $g$ is differentiable at $x^{*} \notin D$, we have

$$
\left(\frac{g}{\psi}\right)^{\prime}\left(x^{*}\right)=0,
$$

which is equivalent to $V^{\prime}\left(x^{*}\right)=g^{\prime}\left(x^{*}\right)$. 
In the next proposition we establish a stronger result, which contains the previous one. Let us recall the following definition from [21].

Definition 4.1. A real number $b$ is a left boundary of the stopping region $E$ if, for small enough $\varepsilon,[b, b+\varepsilon]$ belongs to $E$ and $(b-\varepsilon, b)$ belongs to the continuation region. A real number $b$ is a right boundary of the stopping region $E$ if, for small enough $\varepsilon,[b-\varepsilon, b]$ belongs to $E$ and $(b, b+\varepsilon)$ belongs to the continuation region.

Proposition 4.2. Assume that $g$ satisfies Assumption 2.2. Let $b$ be a boundary of $E$ such that $b \notin D$. Then $V$ is differentiable at $b$ with $V^{\prime}(b)=g^{\prime}(b)$.

Proof. Without loss of generality, we give the proof only for the case of a right boundary. For $x>b$, we have

$$
\frac{V(x)-V(b)}{x-b} \geq \frac{g(x)-g(b)}{x-b} .
$$

Therefore, $D_{+} V(b):=\lim _{h \rightarrow 0^{+}}(V(b+h)-V(b)) / h \geq g^{\prime}(b)$. By definition, $[b-\varepsilon, b]$ belongs to $E$ for small enough $\varepsilon$. Therefore, for $b-\varepsilon<x<b$ we have

$$
\frac{V(b)-V(x)}{b-x}=\frac{g(b)-g(x)}{b-x},
$$

which implies that $D_{-} V(b)=g^{\prime}(b)$.

Assume that $D_{+} V(b)>D_{-} V(b)$. Then the Itô-Tanaka formula and the similar local-time argument used in Lemma 4.1 yield

$$
\begin{aligned}
\mathrm{E}_{b}\left(\mathrm{e}^{-r t} V\left(X_{t}\right)\right) & =V(b)+\frac{1}{2}\left(D_{+} V(b)-D_{-} V(b)\right) \mathrm{E}_{b}\left(L_{t}^{b}\right)+o(\sqrt{t}) \\
& >V(b),
\end{aligned}
$$

for small enough $t$. Therefore, the assumption that $D_{+} V(b)>D_{-} V(b)$ contradicts the supermartingale property of $\left(\mathrm{e}^{-r t} V\left(X_{t}\right)\right)_{t \geq 0}$. Thus, the smooth-fit principle applies at $b$.

\subsection{A remark on the propagation of convexity}

We close this section with an example which proves that the value function associated with a convex payoff function is not necessarily convex when the underlying process follows the stochastic differential equation (2.1). We recall that the convexity of the value function and its connection with volatility misspecification has recently been studied in depth; see [9], [18], [11], [7], and [1]. A general result comes from these papers: the value function is convex if either

- $\delta$ is constant, that is, $\left(\mathrm{e}^{-(r-\delta) t} X_{t}\right)_{t \geq 0}$ is a local martingale [9], [18], [11], [7], or

- $x \delta(x)$ is nondecreasing and $\mathrm{e}^{-r t} X_{t}$ converges to 0 in $L^{1}$ (see [1, Corollary 1]).

Consider a one-dimensional diffusion of type (2.1) with $\delta(x)=\delta \mathbf{1}_{\{x \leq a\}}, \sigma(x)=\sigma$, and the optimal stopping problem

$$
V(x)=\sup _{\tau \in \mathcal{T}_{0, \infty}} \mathrm{E}\left(\mathrm{e}^{-r \tau}\left(X_{\tau}-1\right)\right)
$$

Note that $\delta$ is not continuous but satisfies the local integrability condition (2.2). Moreover, we have $V(0)=0$ and $V(x) \leq x$ for every $x>0$. We will prove that a threshold strategy is not optimal. 
The measure $\mathcal{A} g$ is absolutely continuous with respect to the Lebesgue measure $\mathcal{A} g(\mathrm{~d} x)=$ $\mathscr{A} g(x) \mathrm{d} x$ with

$$
\mathscr{A} g(x)=(r-\delta x) \mathbf{1}_{\{x \leq a\}}+r \mathbf{1}_{\{x>a\}} .
$$

Therefore, for $x>a$ we have

$$
V(x) \geq \mathrm{E}_{x}\left(\mathrm{e}^{-r T_{a}} g\left(X_{T_{a}}\right)\right)=g(x)+\mathrm{E}_{x}\left(\int_{0}^{T_{a}} \mathrm{e}^{-r s} r \mathrm{~d} s\right)>g(x) .
$$

We now have to prove that the stopping region is nonempty for some 'good' choice of the parameter $a$. Let us define $\alpha$ and $\beta$ to be the negative and, respectively, positive roots of the equation

$$
\frac{\sigma^{2}}{2} x^{2}+\left(r-\delta-\frac{\sigma^{2}}{2}\right) x-r=0 .
$$

Note that $\beta>1$ and $\alpha+2 r / \sigma^{2}>0$. Fix an $a>\left(2 r+\beta \sigma^{2}\right) /(\beta-1) \sigma^{2}$. The fundamental solutions, $\varphi$ and $\psi$, can be constructed explicitly by matching a linear combination of the fundamental solutions to $\mathcal{A} u=0$ on each of the subintervals $(0, a)$ and $(a, \infty)$. We obtain

$$
\varphi(x)=\left(x^{\alpha}-\frac{2 r+\alpha \sigma^{2}}{2 r+\beta \sigma^{2}} a^{\alpha-\beta} x^{\beta}\right) \mathbf{1}_{\{x \leq a\}}+\frac{\beta-\alpha}{2 r+\beta \sigma^{2}} a^{\alpha+2 r / \sigma^{2}} x^{-2 r / \sigma^{2}} \mathbf{1}_{\{x>a\}}
$$

and

$$
\psi(x)=x^{\beta} \mathbf{1}_{\{x \leq a\}}+\left[\frac{2 r+\beta \sigma^{2}}{2 r+\sigma^{2}} a^{\beta-1} x-\frac{\beta-1}{1+2 r / \sigma^{2}} a^{\beta+2 r / \sigma^{2}} x^{-2 r / \sigma^{2}}\right] \mathbf{1}_{\{x>a\}} .
$$

Note that $\varphi$ is differentiable, strictly decreasing, and convex on $(a, \infty)$ and $\psi$ is differentiable, strictly increasing, and concave on $(a, \infty)$.

Let us assume that $E$ is empty. Therefore, $V(x)=B \psi(x)$ since $V(0)=0$. Moreover, [3, Proposition 5.4] gives

$$
\lim _{x \rightarrow \infty} \frac{V(x)}{\psi(x)}=\lim _{x \rightarrow \infty} \frac{g(x)}{\psi(x)}=\frac{2 r+\sigma^{2}}{2 r+\beta \sigma^{2}} a^{1-\beta}=l_{\infty} .
$$

Thus, $V(x)=l_{\infty} \psi(x)$. However, $V(a)=\left[\left(2 r+\sigma^{2}\right) /\left(2 r+\beta \sigma^{2}\right)\right] a$ and, therefore, $V(a)<$ $a-1$ for $a>\left(2 r+\beta \sigma^{2}\right) /(\beta-1) \sigma^{2}$, which is a contradiction. Finally, $E=\left[\beta /(\beta-1), x_{r}\right]$ is nonempty, with $x_{r} \leq a$. Moreover, $V$ is not globally convex on $(a, \infty)$.

\section{Examples arising in real option theory}

One of the modern applications of optimal stopping theory is to the real option theory of investment under uncertainty. In this framework the optimal investment policy can be mathematically determined as the solution to an optimal stopping problem. The prototype of this approach is the model of [19], in which the underlying value of the investment project evolves as a geometric Brownian motion. In this formulation, the optimal investment strategy is a threshold strategy. Specifically, the investment option should be exercised at the first time the value of the investment project exceeds a critical threshold, the optimal exercise boundary, which can be explicitly computed using a standard verification theorem based on the smooth-fit principle (see also [5, Part III]). Applications of continuous-time real option theory to optimal stopping problems where the optimality of threshold strategies is assumed a priori abound in the current literature. We will discuss some of them below. 


\subsection{Sequential irreversible investment}

Consider a project that can be decomposed into two stages. Once the two stages have been completed, the instantaneous payoff is $\alpha X$, where $X$ satisfies the stochastic differential equation (2.1). We assume that the function $x \delta(x)$ is increasing in such a way that every optimal stopping problem of the type

$$
\sup _{\tau \in \mathcal{T}_{0, \infty}} \mathrm{E}\left(\mathrm{e}^{-r \tau}\left(\alpha X_{\tau}-I\right)\right)=\alpha \sup _{\tau \in \mathcal{T}_{0, \infty}} \mathrm{E}\left(\mathrm{e}^{-r \tau}\left(X_{\tau}-K\right)\right) \quad \text { with } K=\frac{I}{\alpha}
$$

admits an optimal threshold strategy according to Theorem 4.1 and Example 4.1, that is, with $E=\left[x^{*}(K), \infty\right)$. Moreover, Proposition 4.1 implies that $x^{*}$ is a nondecreasing function of $K$. Let $\alpha=\alpha_{1}+\alpha_{2}$ and let $I_{1}$ and $I_{2}$ be positive constants. The optimal stopping problem arising from a sequential investment then has a payoff function given by

$$
g(x)=\alpha_{1} x-I_{1}+F_{2}(x),
$$

where

$$
F_{2}(x)=\sup _{\tau \in \mathcal{T}_{0, \infty}} \mathrm{E}\left(\mathrm{e}^{-r \tau}\left(\alpha_{2} X_{\tau}-I_{2}\right)\right)
$$

Theorem 4.1 yields

$$
F_{2}(x)= \begin{cases}\left(\alpha_{2} x_{2}^{*}-I_{2}\right) \frac{\psi(x)}{\psi\left(x^{*}\right)}, & x \leq x_{2}^{*}, \\ \alpha_{2} x-I_{2}, & x \geq x_{2}^{*}\end{cases}
$$

We assume that

$$
\frac{I_{1}}{\alpha_{1}}<\frac{I_{1}+I_{2}}{\alpha_{1}+\alpha_{2}}<\frac{I_{2}}{\alpha_{2}},
$$

which ensures that the two stages are not accomplished simultaneously. Under this assumption, the measure $\mathcal{A} g=r I_{1}-\alpha_{1} x \delta(x)$ satisfies the assumption of Theorem 4.1 with $x_{1}$ defined by $x_{1} \delta\left(x_{1}\right)=r I_{1} / \alpha_{1}$. Therefore, the associated stopping region is $\left[x_{1}^{*}, \infty\right)$, where $x_{1}^{*}$ is implicitly given by the equation

$$
g(x) \psi^{\prime}(x)-g^{\prime}(x) \psi(x)=0 .
$$

Since, $F_{2}^{\prime} \psi=F_{2} \psi^{\prime}$ on $\left(0, x_{2}^{*}\right)$, we deduce that $x_{1}^{*}$ is characterized by

$$
\left(\alpha_{1} x_{1}^{*}-I_{1}\right) \psi^{\prime}\left(x_{1}^{*}\right)=\alpha_{1} \psi\left(x_{1}^{*}\right) .
$$

Therefore, the sequential investment threshold is equal to the investment threshold of the optimal stopping problem associated with the payoff $\alpha_{1} x-I_{1}$. The option to invest in the second stage has no effect on the investment threshold for the first stage.

\subsection{Investment in a leveraged firm}

Consider an investor who has the opportunity to invest, at a cost $I$, in a firm whose instantaneous cash flow follows a geometric Brownian motion such that

$$
\mathrm{d} X_{t}=X_{t}\left((r-\delta) \mathrm{d} t+\sigma \mathrm{d} W_{t}\right) .
$$

The firm has issued a perpetual debt with a coupon flow $c$ per unit time. It is assumed that bankruptcy occurs when the cash flow is not sufficient to cover the interest payment $c$. That is, the investor has to solve

$$
\sup _{\tau \in \mathcal{T}_{0, \infty}} \mathrm{E}\left(\mathrm{e}^{-r \tau} g\left(X_{\tau}\right)\right)
$$


with

$$
g(x)=\mathrm{E}_{x}\left(\int_{0}^{\tau_{c}} \mathrm{e}^{-r s}\left(X_{s}-c\right) \mathrm{d} s\right)-I,
$$

where $\tau_{c}=\inf \left\{t \geq 0: X_{t} \leq c\right\}$. An easy computation gives

$$
g(x)=\left(\frac{x}{\delta}-\frac{c}{r}-\left(\frac{r-\delta}{r \delta}\right) c\left(\frac{x}{c}\right)^{\alpha}\right) \mathbf{1}_{\{x \geq c\}}-I,
$$

where $\alpha$ is the negative root of the equation

$$
\frac{\sigma^{2}}{2} x^{2}+\left(r-\delta-\frac{\sigma^{2}}{2}\right) x=r .
$$

Therefore, the open set $\{x \geq 0: g(x)>0\}$ is right connected and $\mathcal{A} g=r I+c-x$ holds within it. Hence, a threshold strategy is optimal.

\subsection{Irreversible investment in alternative projects}

We revisit the model of [4] for a state process that satisfies (2.1). Consider an optimal stopping problem with

$$
g(x)=\max \left(\alpha_{1} x-I_{1}, \alpha_{2} x-I_{2}\right), \quad I_{1}<I_{2}, \alpha_{1}<\alpha_{2} .
$$

If we separately consider the respective optimal stopping problems with $g_{i}(x)=\alpha_{i} x-I_{i}, i=$ 1,2 , then standard arguments give the existence of two optimal thresholds $x_{1}^{*}$ and $x_{2}^{*}$. Assuming that

$$
\frac{I_{1}}{\alpha_{1}}<\frac{I_{2}}{\alpha_{2}},
$$

we have $x_{1}^{*} \leq x_{2}^{*}$ by Proposition 4.1. Moreover, Lemma 4.1 shows that $\left(I_{2}-I_{1}\right) /\left(\alpha_{2}-\alpha_{1}\right)$ does not belong to the stopping region. Two cases have to be considered.

- If $x_{1}^{*} \geq\left(I_{2}-I_{1}\right) /\left(\alpha_{2}-\alpha_{1}\right)$ then $E=\left[x_{2}^{*}, \infty\right)$ (a threshold strategy is optimal).

- If $x_{1}^{*}<\left(I_{2}-I_{1}\right) /\left(\alpha_{2}-\alpha_{1}\right)$ then either

(i) $g\left(x_{1}^{*}\right) / \psi\left(x_{1}^{*}\right) \leq g\left(x_{2}^{*}\right) / \psi\left(x_{2}^{*}\right)$, in which case $E=\left[x_{2}^{*}, \infty\right)$ (a threshold strategy is optimal), or

(ii) $g\left(x_{1}^{*}\right) / \psi\left(x_{1}^{*}\right)>g\left(x_{2}^{*}\right) / \psi\left(x_{2}^{*}\right)$, in which case $E=\left[x_{1}^{*}, x_{l}\right] \cup\left[x_{r}, \infty\right)$ (a threshold strategy is not optimal).

In the case in which $X$ follows a geometric Brownian motion as in [4], the computation of the optimal threshold is explicit.

\subsection{Irreversible investment and time to build}

Consider an investor who has the opportunity to invest in a project at a cost $I+J$ at any date $\tau$. He chooses to invest an amount $J$ to undertake the project by issued debt. We assume that the revenue of the project will be returned at time $\tau+T$, where $T$ is the time to build the project. Moreover, the investor has to pay the nominal, $D>0$, of a debt contract at time $\tau+T$. The problem is

$$
\sup _{\tau \in \mathcal{T}_{0, \infty}} \mathrm{E}\left(\mathrm{e}^{-r \tau} g\left(X_{\tau}\right)\right)
$$


with

$$
g(x)=\mathrm{E}\left(\mathrm{e}^{-r T}\left(X_{T}-D\right)_{+}\right)-I=: C(T, x)-I .
$$

If we assume that $X$ follows a geometric Brownian motion, we obtain the Black-Scholes formula,

$$
g(x)=x \mathrm{e}^{-\delta T} \mathcal{N}\left(d_{1}\right)-D \mathrm{e}^{-r T} \mathcal{N}\left(d_{2}\right)-I,
$$

where $\mathcal{N}$ is the distribution function of the standard Gaussian law. Now,

$$
\mathcal{A} g(x)=\mathcal{A} C(T, x)+r I=r I+\partial_{T} C(T, x) .
$$

Straightforward but tedious calculus shows that $\mathcal{A} g$ satisfies the assumptions of Theorem 4.1. Therefore, a threshold strategy is optimal.

\section{Acknowledgements}

I would like to thank Damien Lamberton and Erik Ekström for their helpful comments.

\section{References}

[1] Alvarez, L. R. H. (2003). On the properties of $r$-excessive mappings for a class of diffusions. Ann. Appl. Prob. 13, 1517-1533.

[2] Borodin, A. N. And Salminen, P. (1996). Handbook on Brownian Motion-Facts and Formulae. Birkhäuser, Basel.

[3] Dayanik, S. and Karatzas, I. (2003). On the optimal stopping problem for one-dimensional diffusions. Stoch. Process. Appl. 107, 173-212.

[4] Décamps, J. P., Mariotti, T. and Villeneuve, S. (2006). Irreversible investment in alternative projects. Econometric Theory 28, 425-448.

[5] Dixit, A. K. AND PINDYCK, R. S. (1994). Investment Under Uncertainty. Princeton University Press.

[6] Dupuis, P. AND WANG, H. (2005). On the convergence from discrete to continuous time in an optimal stopping problem. Ann. Appl. Prob. 15, 1339-1366.

[7] Екsтröm, E. (2004). Properties of American option prices. Stoch. Process. Appl. 114, 265-278.

[8] El Karoui, N. (1981). Les aspects probabilistes du contrôle stochastique. In Ninth Saint Flour Prob. Summer School (Lecture Notes Math. 876), Springer, Berlin, pp. 74-239.

[9] El Karoui, N., Jeanblanc, M. and Shreve, S. (1998). Robustness of the Black and Scholes formula. Math. Finance 8, 93-126.

[10] Itô, K. And McKean, H. P., JR. (1965). Diffusion Processes and Their Sample Paths. Springer, Berlin.

[11] Janson, S. And Tysk, J. (2003). Volatility time and properties of option prices. Ann. Appl. Prob. 13, 890-913.

[12] Jönsson, H., Kukush, A. G. And Sylvestrov, D. S. (2005). Threshold structure for optimal stopping strategy for American type option. I. Theory Prob. Math. Statist. 71, 93-103.

[13] Karatzas, I. And Shreve, S. (1988). Brownian Motion and Stochastic Calculus. Springer, New York.

[14] Karatzas, I. And Shreve, S. (1998). Methods of Mathematical Finance. Springer, Berlin.

[15] Kotlow, D. B. (1973). A free boundary problem connected with the optimal stopping problem for diffusion processes. Trans. Amer. Math. Soc. 184, 457-478.

[16] Kyprianou, A. E. And Pistorius, M. R. (2003). Perpetual options and Canadization through fluctuation theory. Ann. Appl. Prob. 13, 1077-1098.

[17] Mandl, P. (1968). Analytical Treatment of One-Dimensional Markov Processes. Springer, New York.

[18] Martini, C. (1992). Propagation of convexity by Markovian and martingalian semigroups. Potential Anal. 10, 133-175.

[19] McDonald, R. And Siegel, D. (1986). The value of waiting to invest. Quart. J. Econom. 101, 707-727.

[20] ØKsendal, B. (1998). Stochastic Differential Equations: An Introduction with Applications, 5th edn. Springer, Berlin.

[21] Salminen, P. (1985). Optimal stopping of one-dimensional diffusions. Math. Nachr. 124, 85-101.

[22] Villeneuve, S. (1999). Exercise regions of American options on several assets. Finance Stoch. 3, $295-322$. 